\title{
Bidirectional ventricular tachycardia secondary to aconite toxicity after ingestion of a Chinese herbal supplement in Canada
}

\author{
Janice Lee, Andrew Czarnecki, Mark S. Hansen, Garry Thomas, \\ Claudia Bucci
}

\begin{abstract}
Introduction: Aconite is commonly used in traditional Chinese medicine but if incorrectly prepared or consumed in excessive doses, can cause significant cardiac toxicity and death. The cardiac sequelae include hypotension, bradycardia, and ventricular arrhythmias, including bidirectional ventricular tachycardia. The diagnosis is made clinically and management is supportive. Case Report: A 44-year-old male was admitted to the hospital post cardiac arrest. It was discovered that he had ingested significant quantities of the Chinese herbal medication "Fu $\mathrm{Zi}$ " or "Aconite" prior to admission. His rhythm on telemetry consisted of monomorphic ventricular tachycardia, bidirectional ventricular tachycardia, and ventricular fibrillation. Repeated cardioversions as well therapy with amiodarone was required. The patient's arrhythmias subsided after 24 hours, but the patient had developed global hypoxic
\end{abstract}

Janice Lee ${ }^{1}$, Andrew Czarnecki ${ }^{2}$, Mark S. Hansen ${ }^{2}$, Garry Thomas $^{3}$, Claudia Bucci ${ }^{4}$

Affiliations: 'Staff Pharmacist, Department of Pharmacy, Sunnybrook Health Sciences Centre, Toronto, Ontario, Canada; ${ }^{2}$ Staff Cardiologist, Division of Cardiology, Sunnybrook Health Sciences Centre, Toronto, Ontario, Canada; ${ }^{3}$ Division of Cardiology, Sunnybrook Health Sciences Centre, Toronto, Ontario, Canada; ${ }^{4}$ Clinical Coordinator, Department of Pharmacy, Sunnybrook Health Sciences Centre, Toronto, Ontario, Canada.

Corresponding Author: Claudia Bucci, Sunnybrook Health Sciences Centre, Room E237, 2075 Bayview Avenue, Toronto, Ontario, Canada, M4N 3M5; Email: Claudia. Bucci@ sunnybrook.ca

Received: 24 April 2018

Accepted: 06 June 2018

Published: 19 July 2018 brain injury and life support was withdrawn. Conclusion: Given the increasing popularity of herbal products and growing Asian populations in North America, practitioners must have greater awareness of the clinical presentation and consequences of aconite toxicity.

Keywords: Aconite, Bidirectional ventricular tachycardia, Fatal overdose

\section{How to cite this article}

Lee J, Czarnecki A, Hansen MS, Thomas G, Bucci C. Bidirectional ventricular tachycardia secondary to aconite toxicity after ingestion of a Chinese herbal supplement in Canada. Int $\mathrm{J}$ Case Rep Images 2018;9:100937Z01JL2018.

Article ID: 100937Z01JL2018

$$
* * * * * * * * *
$$

doi: 10.5348/100937Z01JL2018CR

\section{INTRODUCTION}

Aconite is a traditional Chinese herbal product with the potential to cause cardiac toxicity including complex ventricular arrhythmias such as bidirectional ventricular tachycardia [1]. It is available in a wide variety of dosage forms, such as tablets, capsules, pastes, tinctures, or as the raw root [2]. Consuming the plant directly, or ingesting large quantities, can cause significant cardiac toxicity [2]. The lethal human dose is as little as 1-2 mg, and is associated with a $15 \%$ mortality, often secondary to cardiac arrythmia and heart failure [3]. Aconite is listed in the 2016 American Heart Association Scientific Statement "Drugs that May Cause or Exacerbate Heart Failure" as a product with potentially harmful cardiovascular effects [4]. The increasing use of herbal products and the 


\section{EDORiUM Journals}

growing Asian populations in North America highlight the need for practitioners to be aware of the possibility of aconite toxicity in patients presenting with acute cardiac symptoms [2].

\section{CASE REPORT}

A 44-year-old male of Chinese ethnicity with a history of hypertension and chronic kidney disease (Stage IV) was found unconscious in his home. His initial rhythm was consistent with pulseless electrical activity and cardiopulmonary resuscitation was initiated. Return of spontaneous circulation was achieved with 2 doses of intravenous (IV) epinephrine (1 mg), and he was transferred to hospital. Blood work showed a potassium of $6.1 \mathrm{mEq} / \mathrm{L}$ and a creatinine of $7.7 \mathrm{mg} / \mathrm{dL}$. The patient was administered three doses of IV calcium gluconate ( $1 \mathrm{~g})$. Potassium was shifted with 10 units of IV regular insulin, with $25 \mathrm{~g}$ of $50 \%$ dextrose given prior to prevent hypoglycemia. A bedside echocardiogram revealed normal biventricular systolic function. It was initially suspected that the arrest was secondary to hyperkalemia, and dialysis was initiated. However, the patient remained unstable with incessant ventricular arrhythmias that included bidirectional ventricular tachycardia (VT) (Figure 1).

Further history from the family revealed that he had ingested a large quantity of the Chinese herbal medication "Fu Zi" (Aconite), as he was informed at an Asian market that it was renal protective and may help to avoid dialysis.

During the 24 hours following his presentation, the patient developed periods of bradycardia requiring transcutaneous pacing. He needed multiple vasopressors for hemodynamic support, started prior to arrival at the hospital and titrated to target a mean arterial pressure (MAP) greater than $65 \mathrm{mmHg}$. On arrival, the patient was receiving infusions of norepinephrine at $20 \mathrm{mcg} /$ min, vasopressin at 4 units/hour, and dopamine at 10 $\mathrm{mcg} / \mathrm{kg} / \mathrm{min}$. Dopamine was stopped after two hours, and the patient was transitioned to epinephrine at 20 $\mathrm{mcg} / \mathrm{min}$.Recurrent ventricular arrhythmias included frequent ectopy, monomorphic VT, bidirectional VT, and ventricular fibrillation (VF). The patient was loaded with $300 \mathrm{mg}$ of IV amiodarone followed by an infusion (1

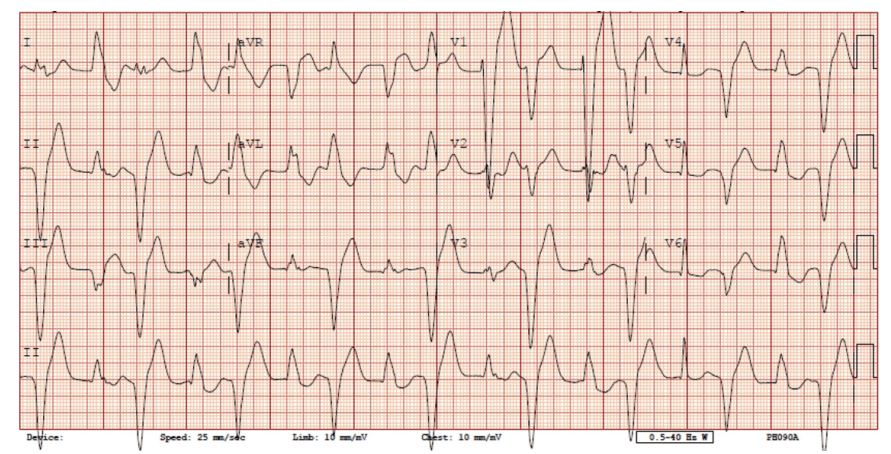

Figure 1: 12-lead ECG in patient with aconite toxicity showing bidirectional ventricular tachycardia. $\mathrm{mg} / \mathrm{min}$ for eight hours then $0.5 \mathrm{mg} / \mathrm{min}$ for 26 hours). During infusion of IV amiodarone, monomorphic VT resolved while bidirectional VT did not, with numerous episodes requiring electrical cardioversion. The local poison control centre was contacted, who suggested administration of the digoxin-specific antibody, as the ECG changes were similar to that of digoxin toxicity. As well, there have been previously reported cases of digitalis mixed with other Chinese herbal medications. Based on this recommendation, 10 vials of $38 \mathrm{mg}$ of the antidote were administered. However, a digoxin level was undetectable.By the following day, his arrhythmias had resolved completely and his hemodynamic status improved. Epinephrine was weaned off after 24 hours, but vasopressin and norepinephrine were continued for 72 hours. Unfortunately, he had developed severe global hypoxic brain injury in consequence to prolonged cardiopulmonary resuscitation and the family ultimately decided to withdraw care.

\section{DISCUSSION}

Aconite is derived from the Aconitum genus, and is common in traditional Chinese medicine as an analgesic, anti-inflammatory, and cardiotonic [5]. The patient was advised to use aconite as a renal protectant, although this has not been previously reported as a common use. Boiling and soaking the product is recommended in order to convert the toxic alkaloids into more benign byproducts, however our patient had forgone this important step in hopes that direct consumption would have more potent medicinal effects [2].

Aconite poisoning is well documented in the Chinese literature, often related to inadequate processing (preboiling) or overdose [2]. Despite the increasing popularity of herbal products in Western societies, little has been reported in North America, and has previously been associated only with accidental ingestion of wild plants [1].

The mechanism of aconite toxicity is due to its high affinity for voltage-sensitive sodium channels, resulting in persistent activation [3]. The cardiovascular manifestations include hypotension, bradycardia, sinus tachycardia, and VT/VF, with arrhythmias occurring most frequently in the first 24 hours [2]. Patients may also present with gastrointestinal (nausea and vomiting, abdominal pain) or neurologic (paresthesias including numbing of face then limbs) symptoms [2].

Bidirectional VT is a rare arrhythmia usually associated with digoxin toxicity or familial catecholaminergic polymorphic VT [6]. In a review of 72 cases of bidirectional VT, digoxin was implicated in $82 \%$ of patients [7]. One group reported the presence of this arrhythmia in myocardial infarction [8]. However, aconite poisoning can also cause bidirectional VT and should be included in the differential diagnosis when an unstable patient presents with this arrhythmia $[1,6,9]$. 


\section{EDORiUM Journals}

The diagnosis of aconite toxicity requires a high level of clinical suspicion, as there are no readily available assays in North America for its detection. Management is generally supportive, as there is no known antidote, and due to its lipid solubility and large molecular size, dialysis is ineffective [2]. Vasopressors and inotropes are recommended for severe hypotension and atropine or pacing for clinically significant bradycardia [3]. Ventricular arrhythmias are often refractory to antiarrhythmic medications, but amiodarone and flecainide have been used and are recommended as first-line therapies [3]. Although electrical cardioversion is often necessary, previous studies have shown that it is often ineffective [2]. This may be explained by the fact that defibrillation halts automaticity by recruiting inactive sodium channels, while aconite toxicity already establishes persistent channel activity [3]. Finally, if patients are persistently unstable, early use of mechanical circulatory support should be considered [2].

\section{CONCLUSION}

Many patients view herbal supplements as safer alternatives to conventional medication because they are derived from natural sources. However, our case highlights the need for caution, as this increasingly common herbal supplement is associated with significant toxicity. Moreover, given the growing prevalence of Chinese immigrants in North America, clinicians need to be aware of the clinical presentation associated with aconite toxicity and the expanded differential diagnosis for bidirectional VT.

\section{REFERENCES}

1. Smith SW, Shah RR, Hunt JL, Herzog CA. Bidirectional ventricular tachycardia resulting from herbal aconite poisoning. Ann Emerg Med 2005 Jan;45(1):100-1.

2. Chan TY. Aconite poisoning. Clin Toxicol (Phila) 2009 Apr;47(4):279-85.

3. Coulson JM, Caparrotta TM, Thompson JP. The management of ventricular dysrhythmia in aconite poisoning. Clin Toxicol (Phila) 2017 Jun;55(5):31321.

4. Page RL 2nd, O’Bryant CL, Cheng D, et al. Drugs that may cause or exacerbate heart failure: A scientific statement from the American heart association. Circulation 2016 Aug 9;134(6):e32-69.

5. Lowe L, Matteucci MJ, Schneir AB. Herbal aconite tea and refractory ventricular tachycardia. N Engl J Med 2005 Oct 6;353(14):1532.

6. Vo KT, Tabas JA, Smollin CG. Alternating ventricular complexes after overdose from an herbal medication. JAMA Intern Med 2017 Aug 1;177(8):1199-201.
7. Cohen SI, Deisseroth A, Hecht HS. Infra-His bundle origin of bidirectional tachycardia. Circulation 1973 Jun;47(6):1260-6.

8. Wase A, Masood AM, Garikipati NV, Mufti O, Kabir A. Bidirectional ventricular tachycardia with myocardial infarction: A case report with insight on mechanism and treatment. Indian Heart J 2014 JulAug;66(4):466-9.

9. Zhao YT, Wang L, Yi Z. An unusual etiology for bidirectional ventricular tachycardia. Can J Cardiol 2016 Mar;32(3):395.e5-6.

$* * * * * * * * *$

\section{Author Contributions}

Janice Lee - Substantial contributions to conception and design, Acquisition of data, Analysis and interpretation of data, Drafting the article, Revising it critically for important intellectual content, Final approval of the version to be published

Andrew Czarnecki - Analysis and interpretation of data, Revising it critically for important intellectual content, Final approval of the version to be published

Mark S. Hansen - Analysis and interpretation of data, Revising it critically for important intellectual content, Final approval of the version to be published

Garry Thomas - Analysis and interpretation of data, Revising it critically for important intellectual content, Final approval of the version to be published

Claudia Bucci - Analysis and interpretation of data, Revising it critically for important intellectual content, Final approval of the version to be published

\section{Guarantor of Submission}

The corresponding author is the guarantor of submission.

\section{Source of Support \\ None}

\section{Consent Statement}

Written consent not obtained. As per author guidelines, patient consent only required "if any identifying information is included in text or figures". Patient confidentiality maintained in text.

\section{Conflict of Interest}

Authors declare no conflict of interest.

\section{Copyright}

(C) 2018 Janice Lee et al. This article is distributed under the terms of Creative Commons Attribution License which permits unrestricted use, distribution and reproduction in any medium provided the original author(s) and original publisher are properly credited. Please see the copyright policy on the journal website for more information. 
Access full text article on other devices

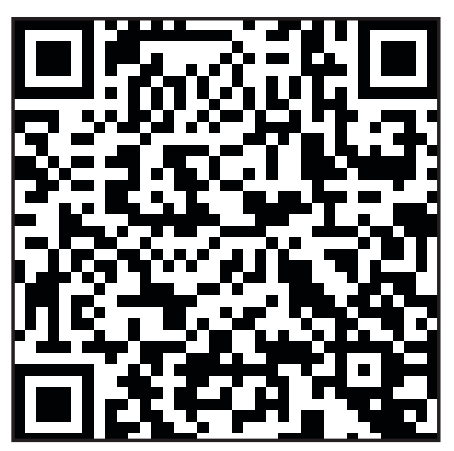

Access PDF of article on other devices

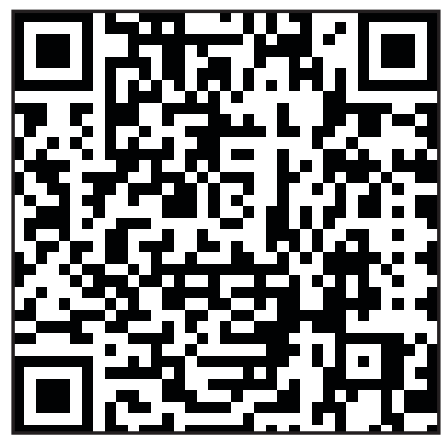




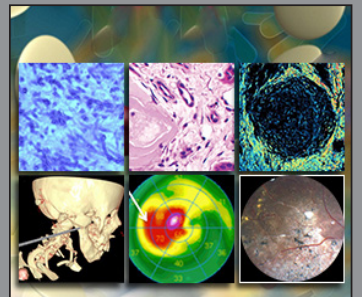

INTERNATIONAL JOURNAL OF CASE REPORTS AND IMAGES
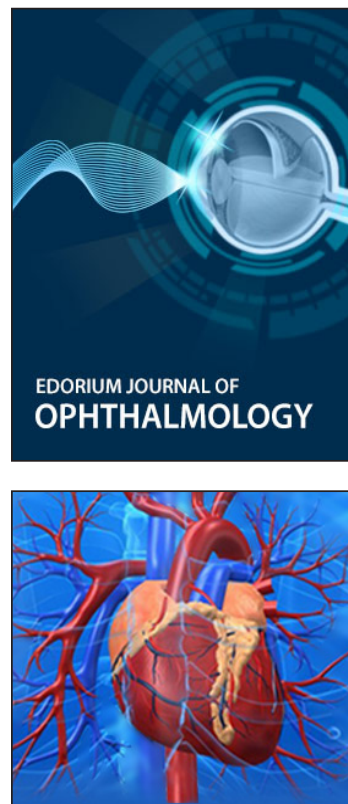

EDORIUM JOURNAL OF

CARDIOTHORACIC AND

VASCULAR SURGERY
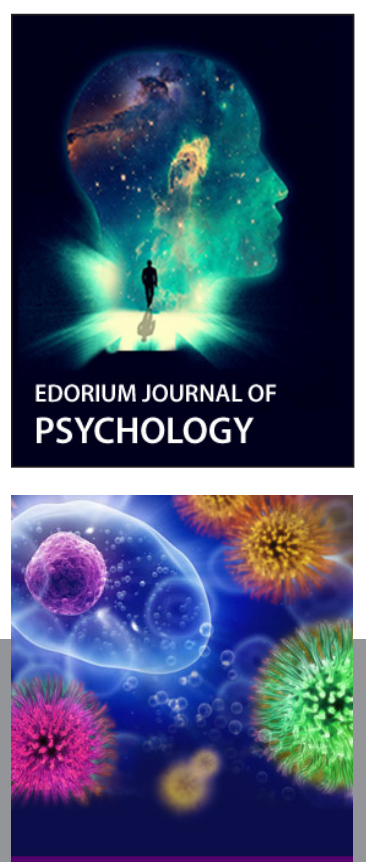

JOURNAL OF CASE REPORTS AND IMAGES IN INFECTIOUS DISEASES
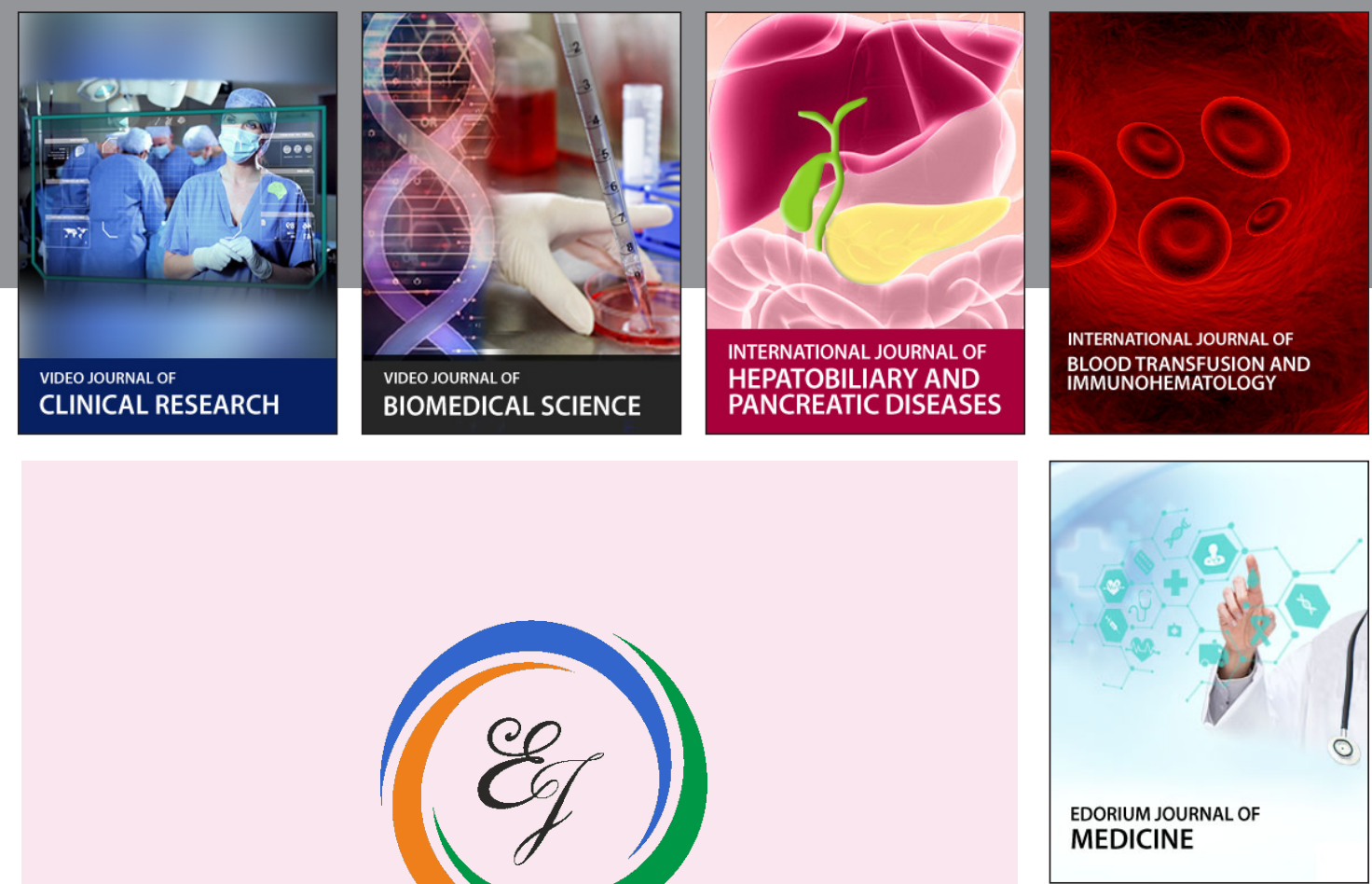

\section{Submit your manuscripts at}

www.edoriumjournals.com
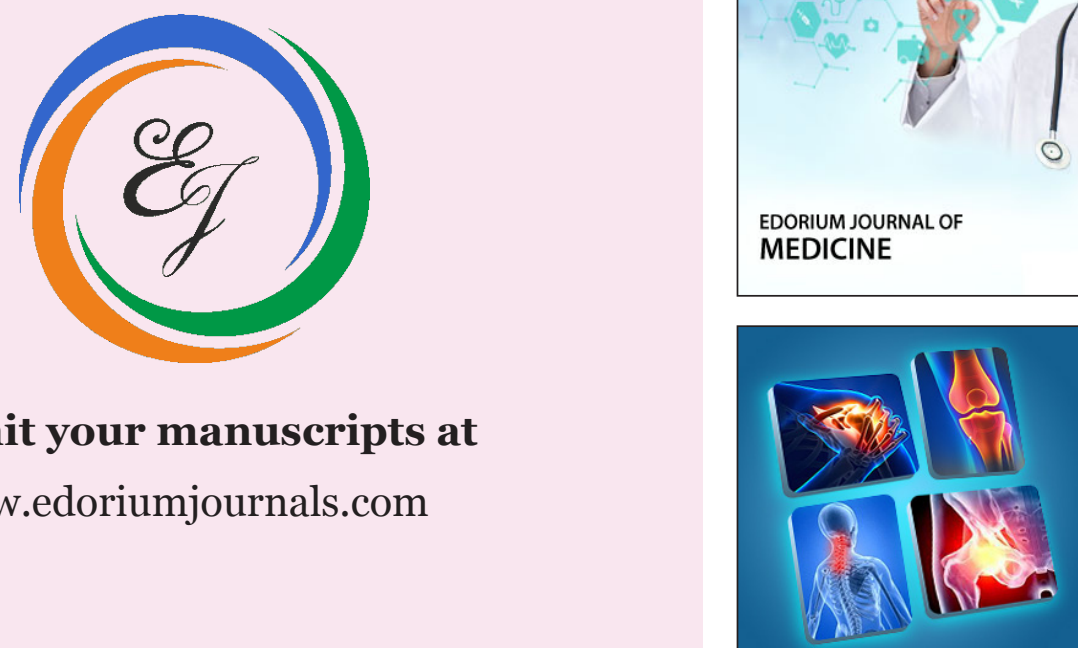

JOURNAL OF CASE REPORTS AND IMAGES IN ORTHOPEDICS AND RHEUMATOLOGY
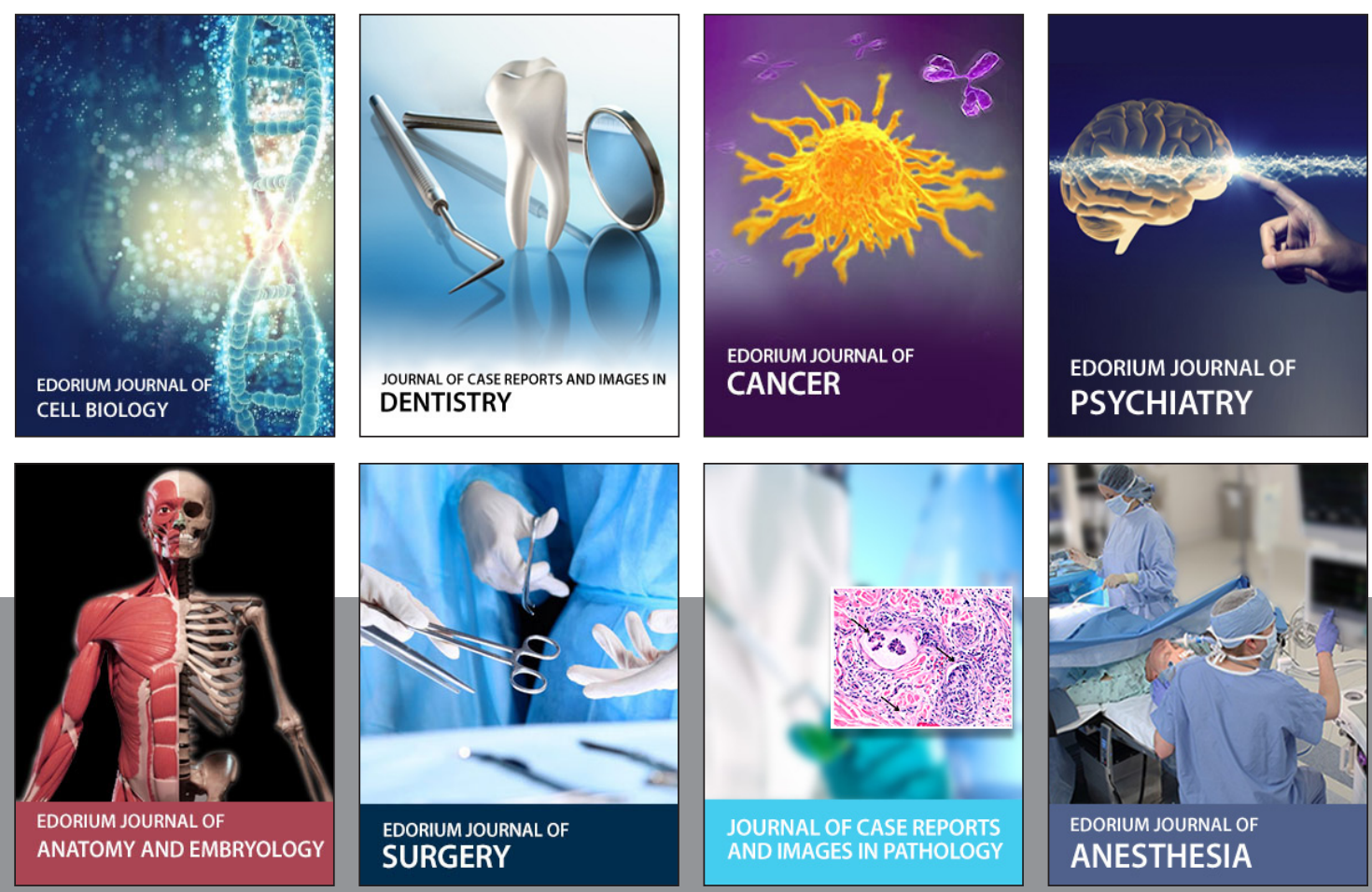